\title{
Studies on Fusarium trichothecene biosynthesis: functional characterization of orthologous pathway genes and development of various types of inhibitors
}

\author{
Kazuyuki Maeda ${ }^{1}$ \\ ${ }^{1}$ Graduate School of Agriculture, Meiji University, 1-1-1 Higashi-Mita, Tama-ku, Kawasaki, Kanagawa 214-8571, Japan
}

\author{
Keywords \\ acivicin; chemical array; \\ Fusarium graminearum; \\ Fusarium sporotrichioides; \\ trichodiene synthase; \\ trichothecene hydroxylase
}

\section{Correspondence}

Kazuyuki Maeda, Graduate School of Agriculture, Meiji University, 1-1-1 Higashi-Mita, Tama-ku, Kawasaki, Kanagawa 214-8571, Japan

E-mail: kmaeda@meiji.ac.jp

(Received February 6, 2018,

accepted April 3, 2018)

\begin{abstract}
Characterization of trichothecene hydroxylation enzymes and screening of trichothecene production inhibitors were performed to decrease the frequency of false detection and reduce grain contamination by trichothecene mycotoxins. Molecular genetic studies of trichothecene biosynthetic genes revealed that the trichothecene C-4 hydroxylase, FgTRI13p, encoded by FgTri13 of Fusarium graminearum, has co-evolved with the C-7/C-8 hydroxylase, FgTRII p, encoded by FgTri1. FgTRI13p shows highly restricted substrate specificity for trichothecene intermediates compared with FsTRI13p produced by Fusarium sporotrichioides. A glutamine analog, acivicin, was screened as an inhibitor of trichothecene production from the chemical library of the RIKEN Natural Products Depository. The predicted mode of action of acivicin in F. graminearum was that the acivicin-induced nutrient starvation repressed the trichothecene master regulator encoded by FgTri6, which blocked trichothecene production. In addition, another trichothecene production inhibitor NPD352 [testosterone 3-(O-carboxymethyl)oxime amide-bonded to phenylalanine methyl ester] was obtained from the library by chemical array screening using trichodiene synthase (FgTRI5p) as a target protein. The mixed-type inhibitor NPD352 may bind and interfere with intracellular FgTRI5p under conditions favoring trichothecene production.
\end{abstract}

\section{Introduction}

Trichothecenes are secondary metabolites produced by Fusarium and other fungal species ${ }^{1)}$. They are characterized by a tetracyclic form of sesquiterpenes including a 9,10-double bond and a 12,13-epoxide ring. The metabolites have various side-chain modification patterns, and more than 200 trichothecenes have been reported ${ }^{2)}$. There are two types of toxic trichothecenes, distinguished based on the presence (type-B) or absence (type-A) of a keto group at C-8 in the trichothecene $A$-ring ${ }^{3)}$. These molecules are also regarded as important contaminants of wheat, barley, maize, and other cereal crops. Most trichothecene contamination, which is responsible for food poisoning, is caused by Fusarium head blight (FHB) pathogen, Fusarium graminearum, and related species ${ }^{4}$. To prevent incorporation of polluted grains into foodstuffs, rigorous monitoring programs are necessary. However, man- agement is difficult because of the multiple chemical properties derived from side-chain modification of the trichothecene skeleton. Therefore, determining the relationships between the function of trichothecene biosynthetic enzymes and side-chain modification patterns may contribute to decreasing the false detection of trichothecene contamination and prediction of undesirable forms of trichothecenes. Similarly, grain contamination may be reduced by identifying and applying trichothecene biosynthesis inhibitors.

This review describes recent insights into sidechain modification mechanisms caused by trichothecene biosynthetic enzymes obtained from molecular genetic studies. Furthermore, two case studies of screening and analysis of the mode of actions of trichothecene production inhibitor are described, which could help establish safe strategies for preventing mycotoxin contamination. 


\section{Evolution of the trichothecene biosynthetic} enzyme: highly restricted substrate specificity for FgTRI13p

Hydroxylation of trichothecene tetracyclic rings ( $\mathrm{A}$, $B, C$, and epoxide-rings) are essential factors that cause various patterns of side-chain modification of Fusarium trichothecene. These hydroxyl groups on the ring are added by trichothecene C-7/C-8 (on A-ring), C-15 (Bring), and $\mathrm{C}-4$ (C-ring) hydroxylases. They are grouped with cytochrome P450 monooxygenase and are expressed as the trichothecene biosynthetic enzymes TRI1p, TRI11p, and TRI13p, respectively. One hydroxylase, TRI1p, functions differently in type-A and B trichothecene producers. Particularly, FsTRI1p of a typical type-A trichothecene-producing species, F. sporotrichioides, introduces hydroxylation at C-8 ${ }^{5)}$, while FgTRI1p of the type-B producer $F$. graminearum introduces hydroxylation at both $\mathrm{C}-7$ and $\mathrm{C}-8^{6}$. After hydroxylation, T-2 toxin, one of the most harmful Fusarium trichothecenes, is biosynthesized in F. sporotrichioides by addition of an isovaleryl group at C-8 (esterification of hydroxyl group) by FsTRI16p ${ }^{7)}$, whereas in F. graminearum, a ketone is added at C-8 to produce deoxynivalenol (DON), nivalenol (NIV), and their acetylated derivatives. These trichothecene metabolites strictly regulate the contamination levels of cereal crops in many countries ${ }^{8)}$. TRI13p, which acts as a C-4 hydroxylase, is present in both $F$. sporotrichioides and as part of an $F$. graminearum species complex. TRI13p has been established as a determining factor of the DON (C-4 deoxy) or NIV (C-4 hydroxy) chemotype of $F$. graminearum ${ }^{9}$. However, its function has not been completely determined, and thus, it is necessary to confirm the difference in substrate specificity for trichothecene intermediates between FsTRI13p and FgTRI13p, focusing on the NIV biosynthetic pathway. Therefore, to gain insight into the $F$. graminearum genes involved in hydroxylation of $C-4$, we examined the activities of Fs/FgTRI13p which modify the trichothecene C-ring by analyzing metabolites and carrying out a feeding assay using transgenic mutants of $F$. graminearum.

To evaluate the substrate specificity of FsTRI13p for C-8 keto-added trichothecene intermediates, thin-layer chromatography (TLC) of the trichothecene product was performed using an FsTri13 transgenic strain of a 3-acetyldeoxynivalenol (3-ADON) producer ${ }^{10)}$. TLC of culture extracts revealed that the FsTri13-expressing strain accumulated 3-acetylnivalenol (3-ANIV), which was hydroxylated at the C-4 position of 3-ADON, in liquid culture (Fig. 1A). Thus, FsTRI13p can also recognize substrates other than the primary substrate, calonectrin (CAL). In contrast, two-dimensional TLC (2D-TLC) of the culture supernatant of the FgTri1 disruption (Fgtri1 ${ }^{-}$) mutant derived from a NIV producing strain MAFF 111233 showed that FgTRI13p has little C-4 hydroxylation activity for $\mathrm{C}-7 / \mathrm{C}-8$ unoxygenated intermediates, CAL, and its deacetylated 3-deacetyl-CAL (Fig. 1B). In addition to analyzing the function of FgTRI13p,
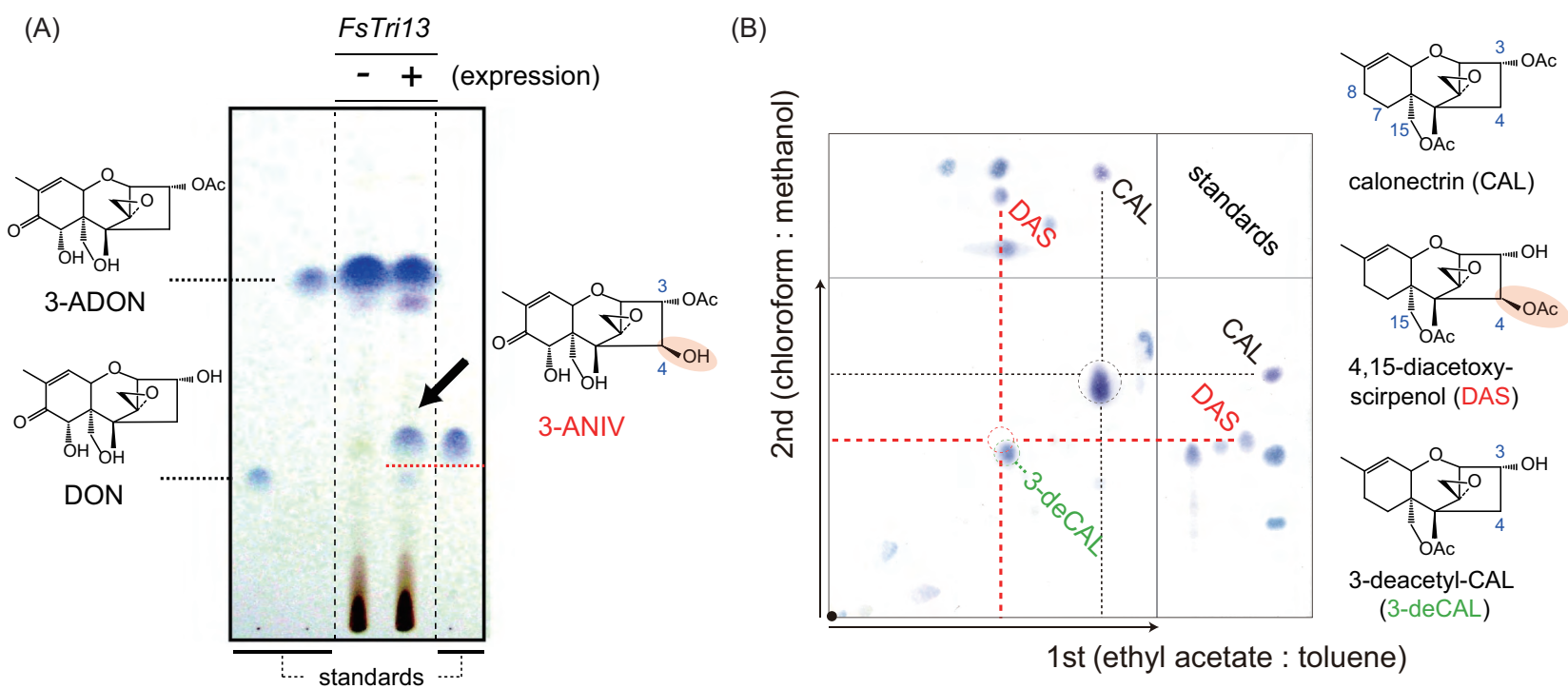

Fig. 1 TLC analyses of trichothecene metabolites from transgenic strains of $F$. graminearum. (A) TLC of culture extracts 4 days after inoculation of 3-ADON producing F. graminearum strain (-) and its transgenic strain expressing FsTri13 (+). Thirty-microgram standards were loaded on the left (DON and 3-ADON) and right (3-ANIV) of the metabolites. (B) 2D-TLC of the culture supernatant of the Fgtri1- mutant at day 7 after inoculation. Trichothecene products equivalent to $2.5 \mathrm{~mL}$ of the culture were spotted on a TLC plate and developed with ethyl acetate:toluene (3:1) in the first dimension, followed by chloroform:methanol (95:5) in the second dimension. Trichothecene standards, CAL $(20 \mu \mathrm{g}), 4,15$-diacetoxyscirpenol (DAS, $20 \mu \mathrm{g})$, and others, were spotted on the top (1st dimension) and right side (2nd dimension). Trichothecenes were detected as colored spots by the NBP/ TEPA reaction as described previously ${ }^{20)}$. 
7-hydroxyisotrichodermin (7-HIT), a C-7 oxygenated trichothecene intermediate, was fed into culture of the Fgtri5 $^{-}$Fgtri1 $^{-}$mutant of strain MAFF 111233 and metabolites were tracked over time by TLC. 7-HIT or its metabolites were not added the C-4 hydroxyl group, demonstrating that FgTRI13p exclusively hydroxylates the regular intermediate, 3,15-diacetyldeoxynivalenol, which was modified at the C-7 hydroxyl and C-8 keto groups of the A-ring (Fig. 2). This substrate specificity indicated that FgTRI13p has evolved more highly restricted substrate specificity against trichothecene intermediates than FsTRI13p. Furthermore, these results suggest that it is necessary to modify the A-ring by FgTRI1p prior to adding the hydroxyl group at C-4 of the C-ring by FgTRI13p ${ }^{11)}$. Thus, co-evolution of FgTRI13p/TRI1p, encoded by FgTri13 and FgTri1, was advantageous for NIV biosynthesis by F. graminearum.

\section{Identification and analyses of mode of actions of trichothecene production inhibitors}

To control FHB, two-types of fungicides, tubulin polymerization inhibitors (e.g., carbendazim and thiophanate-methyl) and sterol demethylation inhibitors (e.g., tebuconazole and metconazole), have been widely applied in the field ${ }^{12}$. However, these fungicides gradually become ineffective in pest management because of the appearance of resistant strains of FHB pathogens ${ }^{13), 14)}$. Additionally, some cases have shown excess regulation levels of trichothecene contamination from non-symptomatic infected grains. Thus, safe and secure fungal growth inhibitors of trichothecene production inhibitors that can be used in combination with the existing fungicides must be developed.

First, we screened inhibitors of the plant cell walldegrading enzyme xylanase which is secreted by F. graminearum and associated with FHB. From the RIKEN Natural Products Depository (NPDepo) library, we identified the glutamine analog acivicin as a candidate based on the decreased halo size using conidia of $F$. graminearum in combination with synthetic minimal agar medium containing remazol brilliant blue xylan as the sole carbon source. However, additional analyses suggested that the effect was related to retardation of conidial germination of the fungus on the medium. Interestingly, application of $25 \mu \mathrm{M}$ acivicin to trichothecene-inducing YS_60 (0.1\% yeast extract, 6\% sucrose) liquid medium significantly reduced 15 -acetyldeoxynivalenol (15-ADON) production by $F$. graminearum strain JCM 9873 without growth inhibition ${ }^{15)}$. Quantitative reverse-transcription PCR (RT-PCR) analyses of the culture mycelia treated with acivicin revealed that the compound repressed expression of the trichothecene biosynthesis master regulator encoded by FgTri6 and

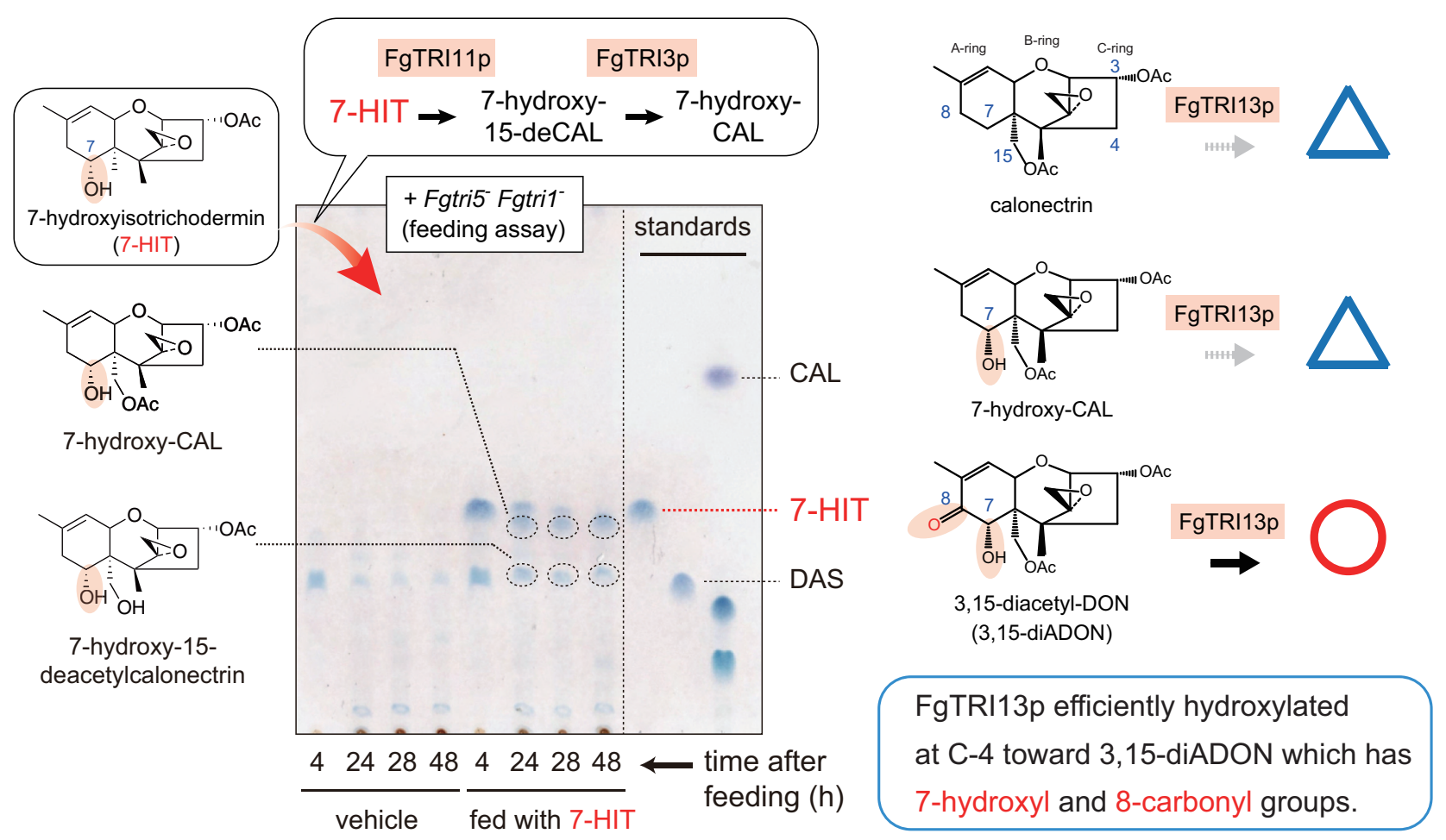

Fig. 2 Analysis of substrate specificity for FgTRI13p by feeding assay. TLC analysis of the metabolites of the Fgtri5 $^{-} \mathrm{Fgtri1}^{-}$mutant at $4,24,28$, and $48 \mathrm{~h}$ after feeding with 7-HIT. The most plausible structures of the major metabolites separated on a TLC plate are indicated. Ethyl acetate:toluene:hexane (19:19:2) was used as a developing solvent. The lanes labeled "vehicle" contain extracts of the mutant culture to which the carrier solvent alone was added. 
downregulated FgTri5 and FgTri4 (Fig. 3A). Furthermore, we evaluated the mode of action of acivicin, for media components which exerted negative effects on acivicin activity by referencing studies in which media compositions affected antibiotic titers ${ }^{16)}$. In the trichothecene production assay, more effective inhibition was observed when synthetic induction medium was used for the main culture rather than YS_60, while the inhibitory effect was completely abrogated when using nutrient abundant complex media for both pre-culture and main culture (Fig. 3B). Thus, for the trichothecene production inhibitory mechanism of acivicin, we estimated that intracellular nutrient starvation induced by acivicin resulted in dysfunction of factors essential for the initial transcription of FgTri6 at the time of trichothecene production by $F$. graminearum ${ }^{17)}$.

Chemical array technology is designed to permit high-throughput screening of beneficial compounds based on the binding activity between the target protein and chemicals immobilized on glass slides ${ }^{18)}$. Some binding chemicals are determined using a specific antibody against a target protein with an affinity tag. Recombinant trichodiene synthase (rTRI5), the initial enzyme in the trichothecene biosynthetic pathway, was used as a target protein for chemical array screening, and 32 candidate chemicals were identified from the chemical library. Although the chemicals did not inhibit trichothecene production, only NPD10133 inhibited rTRI5 activity in vitro. Thus, NPD10133 was used as a parent chemical, and analog screening was performed within the NPDepo library. After screening, 20 analogs of NPD10133 were analyzed to determine the inhibiting activity of trichothecene accumulation in liquid culture. NPD352 [testosterone 3-(O-carboxymethyl)oxime amide-bonded to phenylalanine methyl ester] was identified as a trichothecene production and rTRI5 inhibitor (Fig. 4A). The most efficient inhibitor, NPD352, displayed not only the significant inhibition against $15-A D O N$ produced by $F$. graminearum strain JCM 9873, but also 3-ADON produced by the strain MAFF 101551 at $10 \mu \mathrm{M}$ without growth retardation (Fig. 4B). Although semi-quantitative RT-PCR analyses indicated NPD352 did not decrease Tri expression despite inhibiting trichothecene production ${ }^{19)}$, kinetic
(A)

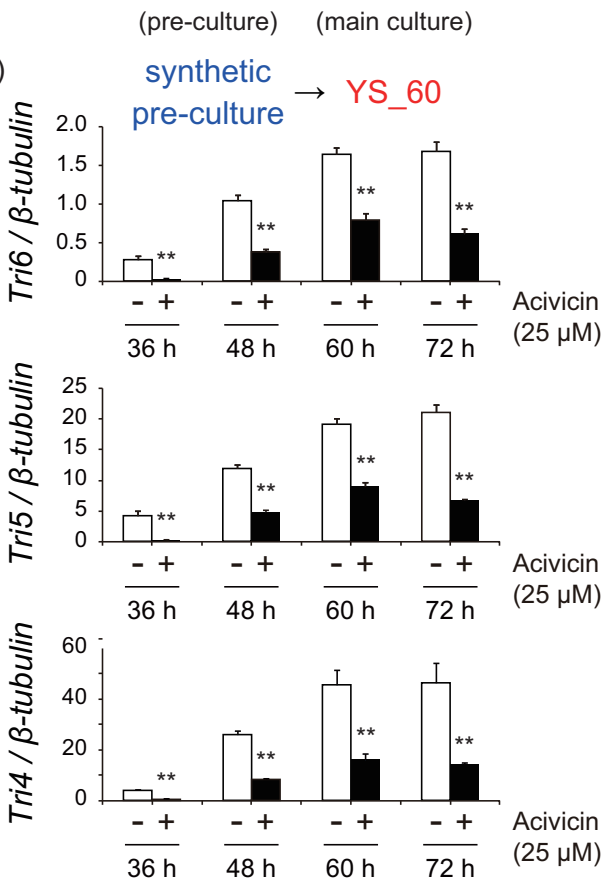

(B)
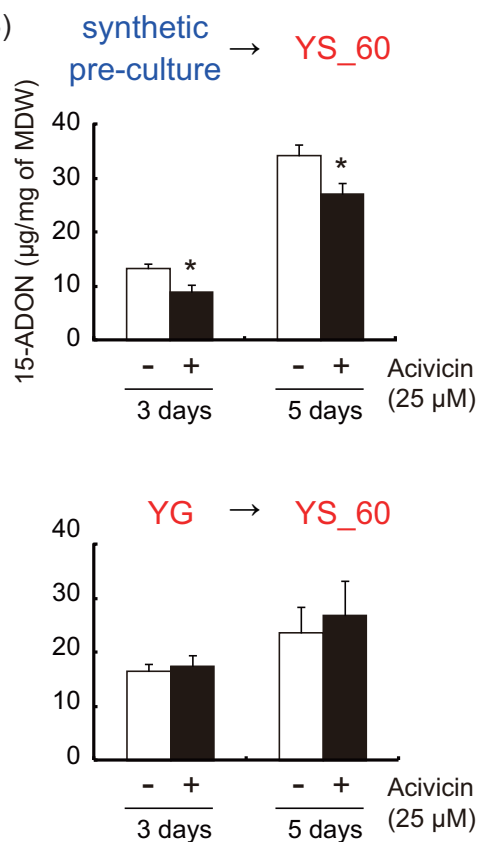

synthetic synthetic pre-culture $\rightarrow$ induction
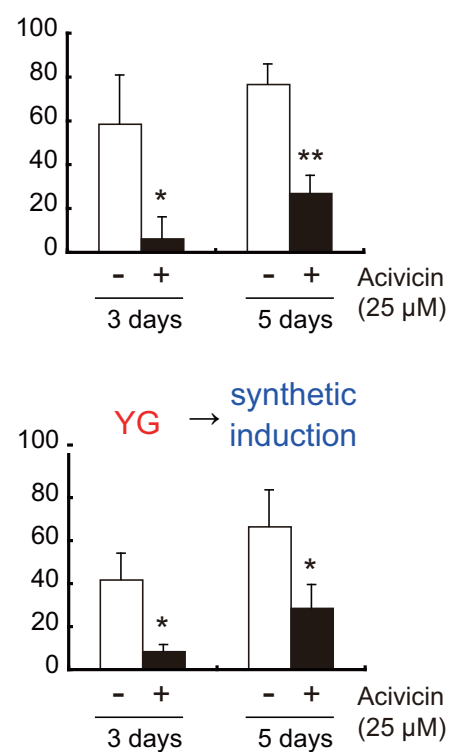

Fig. 3 Characterization of the effects of acivicin on trichothecene production. (A) Effect of acivicin on the expression of Tri6, Tri5, and Tri4. Fusarium graminearum strain JCM 9873 was pre-cultured on synthetic pre-culture medium ${ }^{15)}$ and transferred to YS_60 main culture medium. The mycelia were collected at different time points $(36,48,60$, and $72 \mathrm{~h})$ for RNA extraction. Singlestranded CDNA synthesis and quantitative RT-PCR was performed as described previously ${ }^{17)}$. Expression levels of the Tri genes were normalized to the expression level of $\beta$-tubulin. Acivicin suppressed expression of the Tri genes at all time points examined ${ }^{* *} p<0.01$ as determined by a Student's t-test; $n=3$ ). (B) Effects of acivicin on trichothecene production by different combinations of pre- and main culture media. The strain JCM 9873 was pre-cultured in synthetic pre-culture medium and transferred to YS_60 (upper left) and synthetic induction media ${ }^{17)}$ (upper right), or pre-cultured in YG medium (0.5\% yeast extract, $2 \%$ glucose) followed by transfer to YS_60 (lower left) and synthetic induction media (lower right). Cultures were incubated for 3 and 5 days with or without acivicin $(25 \mu \mathrm{M})$. Toxin contents per $\mathrm{mg}$ of mycelial dry weight (MDW) were calculated and are presented as the means \pm SD. Quantification of 15 -ADON was performed as described previously ${ }^{15)}$. Asterisks denote significant differences $\left({ }^{*} p<0.05,{ }^{* *} p<0.01\right)$ relative to the control without acivicin determined by Student's $t$-test $(n=3)$. Synthetic liquid media (in blue) and complex media (in red) are described over the graphs. 
(A)

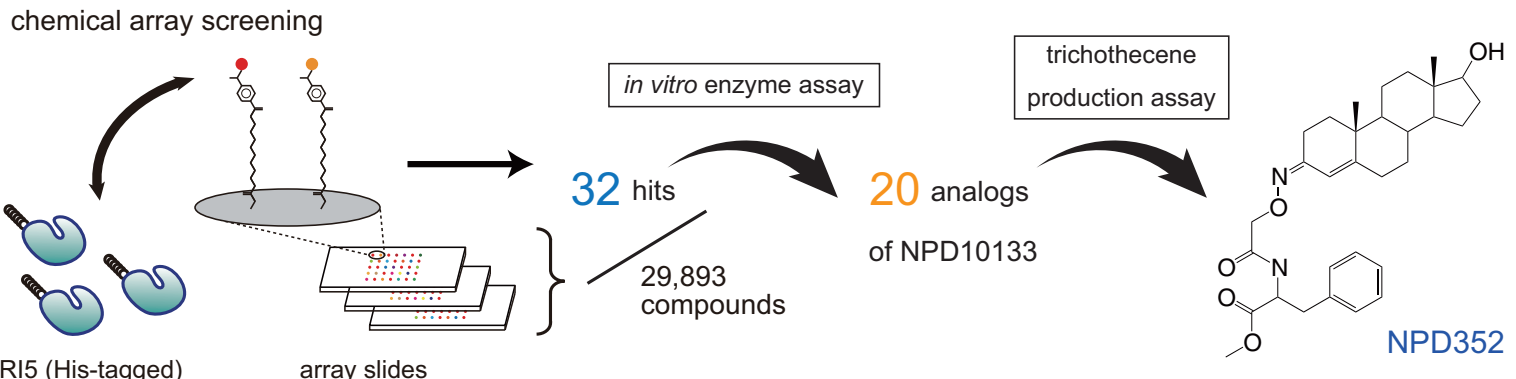

rTRI5 (His-tagged)

array slides

(B)

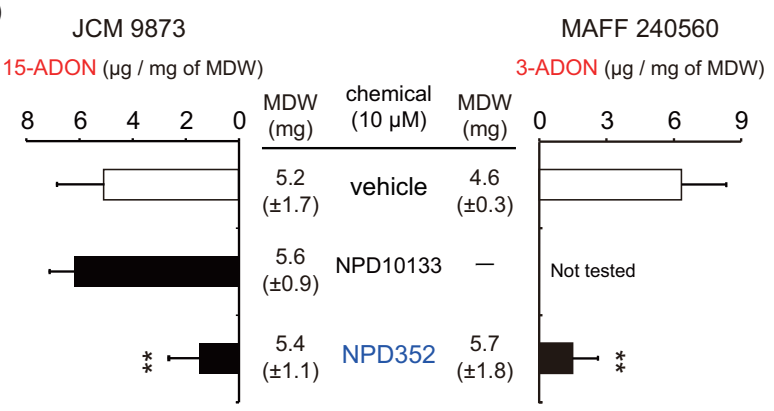

(C)

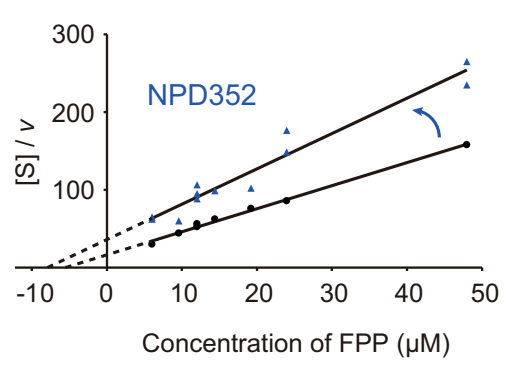

Fig. 4 Evaluation of trichothecene inhibitory effect of trichodiene synthase inhibitor identified by chemical array and library screening. (A) Scheme for chemical array and library screening of trichothecene production inhibitor using trichodiene synthase (TRI5) as a target protein. (B) Effects of NPD10133 and NPD352 for inhibition of trichothecene production by F. graminearum. Strains JCM 9873 and MAFF 240560 were cultured in YS_60 medium containing the carrier solvent 0.1\% (v/v) DMSO (vehicle), 10 $\mu \mathrm{M}$ each of NPD10133 (JCM9873 only), and NPD352 for 2 days. The amounts of trichothecene and mycelial dry weight (MDW) are based on the results of quadruplicate cultures. Asterisks denote a significant difference $\left({ }^{\star *} p<0.01\right)$ relative to the control without an inhibitor (vehicle) as determined by one-way ANOVA, followed by Dunnett's multiple comparison test. (C) Hanes-Woolf plot of rTRI5 inhibition by NPD352. Kinetic parameters with (closed triangle) or without (closed circle) $10 \mu$ M NPD352 were determined from various concentrations of farnesyl pyrophosphate (FPP) substrate. Linear regression was fitted through the plot, whose coefficient of determination $\left(R^{2}\right)$ was calculated as 1.0 (without inhibitor) and 0.95 (with NPD352), respectively.

analysis suggested that NPD352 was a mixed-type inhibitor of rTRI5 (Fig. 4C). Based on the results for NPD352, the inhibitory mechanism for trichothecene production of the compound was caused by binding and inactivation of intracellular FgTRI5p by $F$. graminearum under the conditions of trichothecene production $^{19)}$.

The above findings obtained from basic and applied studies may be useful for overcoming trichothecene contamination. To enhance the feasibility of secure FHB management, the following studies should be conducted: characterization of TRI enzymes from additional Fusarium species; comprehensive analyses using omics for mode of action of the compounds; optimization of the chemical structure for potentiation; and applications in field trials in future studies.

\section{Acknowledgements}

The studies were supported by many researchers and students in the RIKEN, and Meiji, Toyo, and Nagoya Universities. Firstly, I would like to express my great appreciation to Dr. Katsuyoshi Yoneyama for encouraging the work with the RIKEN. I am also grateful to Drs. Akira Tanaka and Naoko Takahashi-Ando of Toyo University for their assistance with LC-MS/MS analysis of trichothecene intermediates, and I am deeply grateful to Dr. Yuichi Nakajima for providing support and suggestions. I appreciate the consideration from Dr. Shuichi Ohsato of Meiji University for providing many helpful suggestions and advice on my life as a researcher. Finally, I express my heartfelt appreciation to Dr. Makoto Kimura of the RIKEN and Nagoya University for providing information regarding genetic engineering of filamentous fungi and for encouraging independence as a researcher of Fusarium graminearum.

\section{References}

1) Kimura, M., Tokai, T., Takahashi-Ando, N., Ohsato, S., Fujimura, M.: Molecular and genetic studies of Fusarium trichothecene biosynthesis: pathways, genes, and evolution. Biosci Biotechnol Biochem, 71, 2105-2123 (2007)

2) Grove, J. F.: The trichothecenes and their biosynthesis. Fortschr Chem Org Naturst, 88, 63-130 (2007) 
3) Ueno, Y.: The toxicology of mycotoxins. Crit Rev Toxicol, 14, 99-132 (1985)

4) Kazan, K., Gardiner, D. M., Manners, J. M.: On the trail of a cereal killer: recent advances in Fusarium graminearum pathogenomics and host resistance. Mol Plant Pathol, 13, 399-413 (2012)

5) Meek, I. B., Peplow, A. W., Ake, C., Jr., Phillips, T. D., Beremand, M. N.: Tril encodes the cytochrome P450 monooxygenase for C-8 hydroxylation during trichothecene biosynthesis in Fusarium sporotrichioides and resides upstream of another new Tri gene. Appl Environ Microbiol, 69, 1607-1613 (2003)

6) McCormick, S. P., Alexander, N. J., Proctor, R. H.: Heterologous expression of two trichothecene P450 genes in Fusarium verticillioides. Can J Microbiol, 52, 220-226 (2006)

7) Peplow, A. W., Meek, I. B., Wiles, M. C., Phillips, T. D., Beremand, M. N.: Tri16 is required for esterification of position C-8 during trichothecene mycotoxin production by Fusarium sporotrichioides. Appl Environ Microbiol, 69, 5935-5940 (2003)

8) Sugita-Konishi, Y.: Toxicity and control of trichothecene mycotoxins. Mycotoxins, 58, 23-28 (2008)

9) Lee, T., Han, Y. K., Kim, K. H., Yun, S. H., Lee, Y. W.: Tri13 and Tri7 determine deoxynivalenol- and nivalenol-producing chemotypes of Gibberella zeae. Appl Environ Microbiol, 68, 2148-2154 (2002)

10) Maeda, K., Tokai, T., Ichikawa, H., Takahashi-Ando, N., Ogura, N., Yoneyama, K., Yoshida, M., Kimura, M.: Production of 3-acetylnivalenol by transgenic Fusarium graminearum expressing Tri13 of type A trichotheceneproducer: participation of the encoded cytochrome P450 monooxygenase in type $\mathrm{B}$ trichothecene biosynthesis. Mycotoxins, 62, 83-90 (2012)

11) Maeda, K., Tanaka, A., Sugiura, R., Koshino, H., Tokai, T., Sato, M., Nakajima, Y., Tanahashi, Y., Kanamaru, K., Kobayashi, T., Nishiuchi, T., Fujimura, M., Takahashi-Ando, N., Kimura, M.: Hydroxylations of trichothecene rings in the biosynthesis of Fusarium trichothecenes: evolution of alternative pathways in the nivalenol chemotype. Environ Microbiol, 18, 3798-3811 (2016)
12) Nakajima, T.: Progress and outlook for the control of nivalenol and deoxynivalenol contamination due to Fusarium head blight in wheat. Mycotoxins, 57, 129-134 (2007)

13) Chen, C. J., Yu, J. J., Bi, C. W., Zhang, Y. N., Xu, J. Q., Wang, J. X., Zhou, M. G.: Mutations in a beta-tubulin confer resistance of Gibberella zeae to benzimidazole fungicides. Phytopathology, 99, 1403-1411 (2009)

14) Yin, Y., Liu, X., Li, B., Ma, Z.: Characterization of sterol demethylation inhibitor-resistant isolates of Fusarium asiaticum and $F$. graminearum collected from wheat in China. Phytopathology, 99, 487-497 (2009)

15) Maeda, K., Nakajima, Y., Motoyama, T., Kitou, Y., Kosaki, T., Saito, T., Nishiuchi, T., Kanamaru, K., Osada, H., Kobayashi, T., Kimura, M.: Effects of acivicin on growth, mycotoxin production and virulence of phytopathogenic fungi. Lett Appl Microbiol, 59, 377-383 (2014)

16) Clavaud, C., Beauvais, A., Barbin, L., Munier-Lehmann, $H_{\text {., }}$ Latge, J. P.: The composition of the culture medium influences the $\beta-1,3$-glucan metabolism of Aspergillus fumigatus and the antifungal activity of inhibitors of $\beta-1,3-$ glucan synthesis. Antimicrob Agents Chemother, 56, 3428-3431 (2012)

17) Maeda, K., Nakajima, Y., Tanahashi, Y., Kosaki, T., Kitou, Y., Kanamaru, K., Kobayashi, T., Nishiuchi, T., Kimura, M.: Characterization of the acivicin effects on trichothecene production by Fusarium graminearum species complex. J Gen Appl Microbiol, 62, 272-276 (2016)

18) Osada, H.: Introduction of new tools for chemical biology research on microbial metabolites. Biosci Biotechnol Biochem, 74, 1135-1140 (2010)

19) Maeda, K., Nakajima, Y., Motoyama, T., Kondoh, Y., Kawamura, T., Kanamaru, K., Ohsato, S., Nishiuchi, T., Yoshida, M., Osada, H., Kobayashi, T., Kimura, M.: Identification of a trichothecene production inhibitor by chemical array and library screening using trichodiene synthase as a target protein. Pestic Biochem Physiol, 138, 1-7 (2017)

20) Takahashi-Ando, N., Tokai, T., Yoshida, M., Fujimura, M., Kimura, M.: An easy method to identify 8-keto-15hydroxytrichothecenes by thin-layer chromatography. Mycotoxins, 58, 115-117 (2008) 